\title{
Some Aspects on Computing Means Development Philosophy
}

\author{
Jaroslav Khetagourov \\ Russian Academy of Sciences, Moscow, Russia \\ tshe@bk.ru
}

\begin{abstract}
Some factors influencing computer facilities and programming development appear in this paper. The negative role of present tendencies and development influence is noted.
\end{abstract}

Keywords: Computer facilities, software development, high-level languages, evaluation reliability, computer viruses.

\section{Introduction}

Computing means (CM) as the unity of computing devices (machines) and programming have been developing in the world for more than a half-century. During this period, scientists achieved fantastic successes in creating computing devices (machines) and in the development of programming.

Computing means have received broadest application in various fields of our life. $\mathrm{CM}$ development experience provides us with many opportunities for analyzing accepted decisions and considering various construction rationality estimations. The last is mainly defined by developing conditions and the aims of CM application.

Let's consider the connection between CM application aims and their development conditions. At the initial stage, CM application sphere was restricted by scientific and (mainly) military aims. That is why there were rather simple estimations concerning the rationality of decision methods. In this situation, we take into account two factors: the time of decision-making and the expenses. Therefore, using of CM in various fields of modern society has been essentially expanded and it has become more complicated. In addition, we have increased requirements to $\mathrm{CM}$ and estimations concerning its application rationality.

\section{Computing Means and Storage Capacity}

The historical development of computing means has shown that it was much simpler to realize the increasing hardware production task than to supply its application areas with programs for problems solving. It has strongly influenced CM development. Developers have begun to improve the basic technical characteristics of computing means (such as speed, memory storage) and productivity of programming (high-level languages, HLL). 
The increase of speed and memory storage capacity of computing devices was mainly provided by elements size reduction, which resulted in increasing its work frequency and reducing the sizes of devices in whole. It was also provided by increasing the number of simultaneously working circuits and computing devices.

The technological methods of increasing the fast-action processors apparently have reached a level of economic rationality. We estimate economic efficiency as the ratio between expenses for technological rigging and the output of a suitable design. On one hand, we can evaluate the market value and expenses for ensuring heat pipe-bend from big integration circuits (BICs) in connection with increasing of work frequency. On the other hand, we can compare the rigging of increased requirements concerning stability of BICs operating conditions for the reliability of the rigging. The increasing number of simultaneously working processors in connection with developing a crystal upon which some processors are placed, has received an additional benefit due to reduction of amount of external communications within the system.

\section{Computing Means and Programming}

The increasing of programming productivity provided by the large-scale application of high-level languages (HLL) takes into consideration the tasks and decision features for various application areas of computing means. The use of high-level languages essentially increases the output of programming tasks. However, the transformation of HLL program into the program for a computing device (i.e. program on the computer language, CL, program) led to substantial growth of its storage volume.

In summarizing the above information, it is possible to characterize the development of computing means as extensive. Accordingly, one could estimate its role in economic development of society. The extensive development of computing means has brightly revealed a positive feedback of its two principal parts: computing devices and programming. Therefore, the increase of program volumes targets an improved performance of computing devices; their perfection leads to the possibility of increasing the software. Thus, these two parts create conditions for each other's development that provides the existing parity in the distribution and development of works. At the same time, it generates economic interests of the sides.

As a result, the concepts of CM development keep the parity of income distribution between manufacturers of hardware and software. The extensive development of computing means negatively influences generation of new ideas and its introduction in $\mathrm{CM}$ construction.

\section{Introspection}

The important problem of computing means development is an estimation of a relationship between types and numbers of operations in HLL and CL and the choice of building computing means. Apparently, it is expedient to carry out analysis of movement from CL to HLL, assuming hardware realization of each step of this movement. The recognized advantages of HLL programming on the one hand, and the achievements in technology of element size minimization on a crystal on the other 
hand, plus the development of CAD tools that give conditions for the practical realization of complicated circuits of operation with HLL.

However, according to publications, this CM direction has not received essential development, apparently because of corporate reasons. On one hand, the marketing of software on CL was sharply limited, and on the other hand, the requirements of highspeed computing devices essentially changed (reduction of the number of CL commands more than an order, with the practical exception of operating systems).

It is necessary to note that application of CM with HLL hardware realization considerably reduces expenses of firms that create systems with computing devices for programming and debugging of programs of an entire system for its operation. However, these are problems for other groups or companies. Another important problem of CM development is the estimation of universality of computing devices for effective calculations of large-scale tasks.

For the overwhelming number of CM users (more than $80 \%$ ), especially for CM working in various fields, the main thing is the minimization of operating cost. We can achieve this in two ways:

1. Application of universal computing devices and typical programs;

2. Application of specialized computing devices that effectively solve certain groups of tasks and apply the most convenient HLL.

The modern level of technology of computing devices, CAD application, creation of basic technical solutions (basing crystals for microprocessors and controllers) changes the assessment of the feasibility toward specialized decisions.

Leading CM manufactures are interested in production of various universal CM that have large series. It keeps extensive development and provides certain profit level and parity between the CM parts. Actually, for CM users it leans toward an increase of expenses. However, the interaction of CM producers is organized in such a way. It is one of the important factors constraining realization of new ideas.

\section{Multi-processing}

The use of crystals with several processors has created favorable conditions to increase the rate of calculations by increasing its number within systems in large-scale quantity. The decision problem to increase the output of many processors system has passed to algorithmers and programmers.

We can estimate the actual speed of system computing means by the ratio between the average of high-speed processor operations and the nominal sum of its action. We estimate the quantity of this ratio by a small number of peculiar properties that we determine by a kind of task and by the skill and intellect of the algorithmers and programmers.

The leading manufacturers of CM hardware and software support this direction; that is, it keeps parity of the income growth for these two groups. To reduce the influence of human factors, it is especially important to consider the direction of the automatic mis-paralleling of programs with the application of associative memory and current structures. (See the work of V. Bourtsev.) 
Despite certain achievements, the problem of CM development regarding the task of ensuring the trustworthiness and reliability of output information continues to remain. As is known, we define trustworthiness of the information (absence of mistakes in output data) by the quality of used control methods of correctness of operating computing devices and the execution of the program by its testing.

It is necessary to note that we define the importance of requirements to trustworthiness of the information by the limited number of CM consumers (15-20\%). However, the damage caused because of erroneous information could reach a quantity of nearly billion units. The way to achieve the requirements regarding $\mathrm{CM}$ trustworthiness and reliability is control. One way applies to hardware control methods (use of odd and even, module 3, codes, a majority method); the other way is applied to programming methods. Currently, hardware control methods extend the majority method by increasing the volume of the equipment and the consumption of $\mathrm{CM}$ energy by a factor of 3.5 to 4 . Due to the use of universal CM, this decision is economically favorable to its producers. The application of programming methods based on the application of universal CM equipment, causes an increase of its performance by a factor of 2.5 to 3 . This appears favorable for the manufacturer of the equipment and for the developer of software.

\section{External Influences}

Well-known methods of control state that neither the volume of equipment nor the increase of speed affects an increase in demand. These data again confirm that the existing economic relations between leading CM manufacturers constrain the creating and introducing new ideas of CM development. From a consumer's point of view, the cause of some incorrect information is the impact of viruses and various defects that cause damage to nearly a billion units.

Let's consider some features of the occurrence of viruses that break the order of program performance of any task. It is necessary to note that the occurrence of viruses within a program has not been connected with the infringement regarding the equipment operations of the computing device. The accepted measures concerning the elimination of a virus influence occur after its detection and they operate only on the virus. To predict the occurrence of a virus just now, according to the published data, is not possible.

In analyzing virus programs, we should note that within them, the commands transform the addresses and change the data in use. A possible number of similar combinations of commands within programs can cause practical harm beyond all bounds. Therefore, existing CM operating procedures are periodically broken by occurrence of the next virus whose influence is eliminated by the creation of the next anti-virus program associated with additional expenses for the user, plus the increasing of the program volume, and demands of speed requirements.

\section{Conclusion}

For elimination of viruses influence, it is necessary to enable the changes of the organization of a computing device and to stipulate the condition of the formation of a 
program. The realization of this direction demands essential processing, organization of work of computing devices, and programming methods that are associated with large expenses.

The economic relations between leading world companies have mainly determined the existing extensive order of $\mathrm{CM}$ development. Such relations constrain the application of basic and new ideas of development of computing systems. This situation can only change because of the appearance of new conditions and social needs. 